\title{
Investigating inequality: a Langevin approach
}

\author{
I. Eliazar]* \\ Smart Device Innovation Science Team, New Devices Group, Intel Corporation, Yakum, Israel
}

Received November 26, 2016

Inequality indices are quantitative scores that gauge the divergence of wealth distributions in human societies from the "ground state" of pure communism. While inequality indices were devised for socioeconomic applications, they are effectively applicable in the context of general non-negative size distributions such as count, length, area, volume, mass, energy, and duration. Inequality indices are commonly based on the notion of Lorenz curves, which implicitly assume the existence of finite means. Consequently, Lorenz-based inequality indices are excluded from the realm of infinite-mean size distributions. In this paper we present an inequality index that is based on an altogether alternative Langevin approach. The Langevin-based inequality index is introduced, explored, and applied to a wide range of non-negative size distributions with both finite and infinite means.

Key words: inequality indices, Lorenz curves, Langevin equation, Gibbs density, scenario-based equality index

PACS: 02.50.-r, 89.65.-S

\section{Introduction}

This paper is written in honor of the $60^{\text {th }}$ birthday of Professor Yurij Holovatch. The scientific interests of Professor Holovatch span from statistical physics to econophysics and sociophysics. In this paper we'll try to weave these interests together via a topic that draws considerable attention among the aforementioned physics audiences: inequality [1] 6$]$.

Following the pioneering work of Vilfredo Pareto on the distribution of wealth in human societies [7], the study of socioeconomic inequality became a major topic in economics and in the social sciences, as well as a major topic of wide public debate [8-26]. Scientists devised special metrics termed inequality indices to gauge socioeconomic inequality [27-30], the most notable of which being the Gini index [31-34] and the Pietra index [35,40]. Inequality indices measure the divergence of wealth distributions from the "ground state" of pure communism, and score this divergence. From an abstract mathematical perspective inequality indices can be applied in the context of general distributions of non-negative sizes - e.g., count, length, area, volume, mass, energy, duration, etc. - and serve, in this general context, as gauges of statistical heterogeneity 41 42].

Inequality indices are based on the notion of Lorenz curves [43,47], which represent wealth distributions - and, in general, distributions of non-negative sizes - in a universally calibrated statistical method. More specifically, inequality indices are based either on geometric divergence [48 49] or on entropic divergence [50 51], from the "ground state" of pure communism, in the space of Lorenz curves. Lorenz curves implicitly require a finite mean of their input distributions. Consequently, inequality indices are applicable only in the case of non-negative size distributions with finite means. However, nonnegative size distributions with infinite means are prevalent across the sciences and are often of prime importance [52-61].

In this paper we propose a new "Langevin approach" to inequality. The approach is based on the Langevin equation [62, 63], a foundational notion in statistical physics that stands at the core of diffusion processes and stochastic dynamics [64, 65]. Following up on the "topography of chance" that emanates

\footnotetext{
*E-mail: iddo.eliazar@intel.com, eliazar@post.tau.ac.il.
} 
from the Langevin equation in the setting of U-shaped potential landscapes [66], we introduce and explore a novel scenario-based inequality index that is applicable for a wide range of non-negative size distributions with both finite and infinite means.

In a sense this paper "closes a circle". Indeed, in [41, 42] it was demonstrated how the socioeconomic methods of Lorenz curves and inequality indices can be "imported" to the physical sciences, and serve there to quantify the statistical heterogeneity of general non-negative size distributions with finite means. This paper moves in the opposite direction, and demonstrates how a Langevin-based approach can effectively replace the Lorenz-based approach of gauging inequality. This is yet another example of the importance of the trans-disciplinary flow of ideas between different fields of science.

The paper is organized as follows. We begin with a brief review of the Langevin equation and its associated Gibbs density (section 2), followed by a brief review of the corresponding geometric Langevin equation and its associated geometric Gibbs density (section 3). After describing the case of underlying Ushaped potential landscapes (section 4, we introduce a scenario-based equality index (sections 5 and 6). We then describe the general application of the scenario-based equality index as a gauge of inequality (section 7), and conclude with illustrative examples of the application (section 8).

\section{Gibbs density}

A key pillar of statistical physics is the notion of entropy maximization: characterizing the most random configuration within a class of configurations that is determined by a given set of constraints [67, 68]. Specifically, consider the class of probability density functions over the real line, $\phi(x)(-\infty<x<\infty)$, that are subject to the following moment constraint: $\int_{-\infty}^{\infty} V(x) \phi(x) \mathrm{d} x=v$, where $V(x)$ is an general moment function, and where $v$ is an admissible moment value. Within this class of densities the unique density that maximizes the Boltzmann-Gibbs-Shannon entropy [69-71] is the following Gibbs density:

$$
\phi(x)=c \exp \left[-\frac{1}{\tau} V(x)\right]
$$

where $c$ is a positive normalization constant, and where $\tau$ is a positive "temperature" parameter that is set to meet the moment constraint.

Yet another key pillar of statistical physics is the Langevin equation: a stochastic differential equation that describes motion on a potential landscape that is subject to random white-noise fluctuations [62 63]. Specifically, consider a potential landscape over the real line $(-\infty<x<\infty)$ that is given by the function $V(x)$, set $F(x)=-V^{\prime}(x)$ to be the corresponding "force function", and denote by $X(t)$ the real-valued position of the motion at time $t$. The stochastic dynamics of the motion are governed by the following Langevin equation:

$$
\dot{X}(t)=F[X(t)]+\sigma \dot{W}(t),
$$

where $\sigma$ is a positive "volatility" parameter, and where $\dot{W}(t)$ is white noise $64,65,1]$

There is a profound connection between these two seemingly unrelated pillars, the notion of entropy maximization and the Langevin equation. Indeed, the stationary law of the motion whose stochastic dynamics are governed by the Langevin equation (2) is given by the Gibbs density of equation (1) with temperature $\tau=\sigma^{2} / 2$. Namely, consider the steady-state position of motion: $X(\infty)=\lim _{t \rightarrow \infty} X(t)$, the limit being in law. Then: the law of the random variable $X(\infty)$ is governed by the Gibbs density of equation (1) with $\tau=\sigma^{2} / 2$.

\section{Geometric Gibbs density}

The Langevin equation (2) describes additive evolution. In many processes, especially in economics and finance, evolution is multiplicative rather than additive [72-76]. The transformation from additive

\footnotetext{
${ }^{1}$ White noise $\dot{W}(t)$ is a stochastic process that is characterized by the following pair of properties [64, 65]: (i) its integral over an arbitrary time interval, $\int_{a}^{b} \dot{W}(t) \mathrm{d} t(a<b)$, is a random variable that is governed by a normal law with mean zero and with variance $b-a$; and (ii) its integrals over disjoint time intervals are independent random variables.
} 
evolution to multiplicative evolution is facilitated by exponentiation. Specifically, in the context of the Langevin equation (2): consider $X(t)$ to be the log-scale position at time $t$, and shift to the position $Y(t)=$ $\exp [X(t)]$. Applying this exponential transformation, Ito's formula [77, 78] implies that the stochastic dynamics of the exponentiated motion are governed by the following geometric Langevin equation:

$$
\frac{\dot{Y}(t)}{Y(t)}=G[Y(t)]+\sigma \dot{W}(t),
$$

where the "geometric force function" is given by $G(y)=\tau+F[\ln (y)](0<y<\infty)$, with $\tau=\sigma^{2} / 2$. Comparing equations (2) and (3) it is indeed evident that the former describes additive evolution, whereas the latter describes multiplicative evolution.

In turn, the stationary law of the motion whose stochastic dynamics are governed by the geometric Langevin equation (3) is given by the following geometric Gibbs density:

$$
\psi(y)=c \exp \left\{-\frac{1}{\tau} V[\ln (y)]\right\} \frac{1}{y}
$$

$(0<y<\infty)$, with temperature $\tau=\sigma^{2} / 2$. Namely, consider the steady-state position of the exponentiated motion: $Y(\infty)=\lim _{t \rightarrow \infty} Y(t)$, the limit being in law. Then: the law of the random variable $Y(\infty)$ is governed by the geometric Gibbs density of equation (4) with $\tau=\sigma^{2} / 2$.

\section{Shapes}

One of the most fundamental potential landscapes, in the context of the Langevin equation, is that of a $U$-shaped "potential well” [66]: $V(x)$ being a convex function with a unique global minimum. The corresponding force function admits the following shape: $F(x)$ is a continuous function that decreases monotonously from a positive limit $F(-\infty):=\lim _{x \rightarrow-\infty} F(x)>0$ to a negative limit $F(\infty):=\lim _{x \rightarrow \infty} F(x)<0$. In what follows we consider this U-shaped potential landscape to hold, and denote by $F^{-1}(\cdot)$ the inverse of the corresponding monotonously decreasing force function.

The stationary Gibbs density $\phi(x)$ of the Langevin equation (2) admits the following unimodal shape: it is monotonously increasing on the half-line $-\infty<x<F^{-1}(0)$, it attains its unique global maximum at the point $F^{-1}(0)$, and it is monotonously decreasing on the half-line $F^{-1}(0)<x<\infty$. Hence, the mode $x_{*}$ of the stationary Gibbs density $\phi(x)$ is the point at which the force function $F(x)$ crosses the level zero: $x_{*}=F^{-1}(0)$. The core of the Langevin equation (2) is the ordinary differential equation $\dot{x}(t)=F[x(t)]$. The stationary solution of this ordinary differential equation, $x(\infty)=\lim _{t \rightarrow \infty} x(t)$, is its fixed point i.e., the point at which the force function $F(x)$ crosses the level zero: $x(\infty)=F^{-1}(0)$. Thus, we observe that the mode of the stationary Gibbs density $\phi(x)$ coincides with the stationary solution of the ordinary differential equation $\dot{x}(t)=F[x(t)]$, i.e.: $x_{*}=x(\infty)$. We shall now argue that the transition from the additive evolution of the Langevin equation (2) to the multiplicative evolution of the geometric Langevin equation (3) breaks this coincidence.

If $F(-\infty) \leqslant \tau$, then the stationary geometric Gibbs density $\psi(y)$ of the geometric Langevin equation (3) is monotonously decreasing on the positive half-line $0<y<\infty$. On the other hand, if $F(-\infty)>\tau$, then the stationary geometric Gibbs density $\psi(y)$ admits the following unimodal shape: it is monotonously increasing on the interval $0<y<\exp \left[F^{-1}(\tau)\right]$, it attains its unique global maximum at the point $\exp \left[F^{-1}(\tau)\right]$, and it is monotonously decreasing on the half-line $\exp \left[F^{-1}(\tau)\right]<y<\infty$. Hence, the mode of the stationary geometric Gibbs density $\psi(y)$ is given by

$$
y_{*}=\left\{\begin{array}{lll}
0, & \text { if } & F(-\infty) \leqslant \tau, \\
\exp \left[F^{-1}(\tau)\right], & \text { if } & F(-\infty)>\tau .
\end{array}\right.
$$

The core of the geometric Langevin equation (3) is the ordinary differential equation $\dot{y}(t)=G[y(t)]$. If $F(\infty)<-\tau$, then the stationary solution of this ordinary differential equation, $y(\infty)=\lim _{t \rightarrow \infty} y(t)$, is its fixed point - i.e., the point at which the geometric force function $G(y)$ crosses the level zero: $y(\infty)=$ $G^{-1}(0)$; note that $G^{-1}(0)=\exp \left[F^{-1}(-\tau)\right]$. On the other hand, if $F(\infty) \geqslant-\tau$, then the stationary solution 
diverges: $y(\infty)=\infty$. Hence, the stationary solution of the ordinary differential equation $\dot{y}(t)=G[y(t)]$ is given by

$$
y(\infty)=\left\{\begin{array}{lll}
\exp \left[F^{-1}(-\tau)\right], & \text { if } & F(\infty)<-\tau, \\
\infty, & \text { if } & F(\infty) \geqslant-\tau .
\end{array}\right.
$$

It is evident from equations (5) and (6) that the mode of the stationary geometric Gibbs density $\psi(y)$ is always smaller than the stationary solution of the ordinary differential equation $\dot{y}(t)=G[y(t)]$, i.e.: $y_{*}<y(\infty)$. Thus, as claimed above, the transition from the additive evolution of the Langevin equation (2) to the multiplicative evolution of the geometric Langevin equation (3) indeed breaks the coincidence of the mode and the stationary solution: we shift from $x_{*}=x(\infty)$ to $y_{*}<y(\infty)$.

\section{Equality: Langevin representation}

An equality index $\mathscr{E}$ is a gauge of positive-valued random variables that scores their inherent equality, i.e., their inherent statistical homogeneity [50 [51]. Equality indices take values in the unit interval, $0 \leqslant$ $\mathscr{E} \leqslant 1$, yield the maximal unit score only in the case of constant random variables, and are scale-invariant (we shall elaborate on the scale-invariance property in section 7 below).

In the context of the Langevin equation (2) and of the geometric Langevin equation (3), the respective steady-state random variables $X(\infty)$ and $Y(\infty)$ are constant only when no randomness is present. The absence of randomness is attained by setting the volatility, and hence the temperature, to vanish: $\tau=$ $\sigma^{2} / 2=0$. Using the notation of the previous section, zero temperature implies that $X(\infty)=x(\infty)$ and $Y(\infty)=\exp [x(\infty)]$. Focusing on the geometric Langevin equation (3), the notation of the previous section further implies that

$$
0 \leqslant y_{*}<\exp \left[F^{-1}(0)\right]<y(\infty) \leqslant \infty,
$$

where:

- $y_{*}$ is the mode of the stationary geometric Gibbs density $\psi(y)$, and is given by equation (5).

- $\exp \left[F^{-1}(0)\right]$ is the constant value of the steady-state random variable $Y(\infty)$, attained at zero temperature.

- $y(\infty)$ is the stationary solution of the ordinary differential equation $\dot{y}(t)=G[y(t)]$, and is given by equation (6).

Equation (7) holds for any positive temperature, $\tau>0$. When the temperature vanishes, $\tau=0$, the strict inequalities of equation (7) become equalities: $y_{*}=\exp \left[F^{-1}(0)\right]=y(\infty)$. Thus, we can use the divergence of the three terms of equation (7) from each other in order to gauge the inherent equality of the steady-state random variable $Y(\infty)$. Indeed, each of the three following ratios can serve as an equality index:

- The "left-hand ratio" $\mathscr{R}_{l}:=y_{*} / \exp \left[F^{-1}(0)\right]$, which vanishes when $F(-\infty) \leqslant \tau$.

- The "right-hand ratio" $\mathscr{R}_{r}:=\exp \left[F^{-1}(0)\right] / y(\infty)$, which vanishes when $F(\infty) \geqslant-\tau$.

- The "overall ratio" $\mathscr{R}:=y_{*} / y(\infty)$, which vanishes when either $F(-\infty) \leqslant \tau$ or $F(\infty) \geqslant-\tau$.

We combine these three ratios together into a joint scenario-based equality index $\mathscr{E}$ that is presented in table 1. Equations (5) and (6) imply the ratio values that are specified in table 1 for each scenario.

\section{Equality: Density representation}

In the previous section we introduced the scenario-based equality index $\mathscr{E}$, and represented it in terms of the underlying Langevin-equation structure: the force function $F(x)$ and the temperature $\tau=$ 
Table 1. The scenario-based equality index $\mathscr{E}$. The rows indicate whether the force-function limit $F(-\infty):=\lim _{x \rightarrow-\infty} F(x)$ is greater than the temperature $\tau$, or not. The columns indicate whether the force-function limit $F(\infty):=\lim _{x \rightarrow \infty} F(x)$ is smaller than minus the temperature $-\tau$, or not. The two rows and the two columns determine four different scenarios, and for each scenario the equality index $\mathscr{E}$ is specified: the ratio $\mathscr{R}$, the ratio $\mathscr{R}_{l}$, the ratio $\mathscr{R}_{r}$, or zero.

\begin{tabular}{|l|c|c|}
\hline \hline & $F(\infty)<-\tau$ & $F(\infty) \geqslant-\tau$ \\
\hline$F(-\infty)>\tau$ & $\mathscr{R}=\exp \left[F^{-1}(\tau)-F^{-1}(-\tau)\right]$ & $\mathscr{R}_{l}=\exp \left[F^{-1}(\tau)-F^{-1}(0)\right]$ \\
\hline$F(-\infty) \leqslant \tau$ & $\mathscr{R}_{r}=\exp \left[F^{-1}(0)-F^{-1}(-\tau)\right]$ & 0 \\
\hline
\end{tabular}

$\sigma^{2} / 2$. This representation is applicable when the underlying Langevin-equation structure is known. However, if this underlying structure is unobservable, and only the stationary geometric Gibbs density $\psi(y)$ is observable, an alternative representation is required. To that end, we introduce the function

$$
\varphi(y)=y \frac{\psi^{\prime}(y)}{\psi(y)}
$$

$(0<y<\infty)$, and note that in terms of the function $\varphi(y)$ the Langevin force function is given by:

$$
\frac{1}{\tau} F(x)=1+\varphi[\exp (x)]
$$

$(-\infty<x<\infty)$. Consequently, the Langevin-based representation of the scenario-based equality index $\mathscr{E}$ — which was specified in table 1 — shifts to a density-based representation. Namely, table 2 specifies the representation of the scenario-based equality index $\mathscr{E}$ in terms of the function $\varphi(y)$ of equation (8).

Table 2. The scenario-based equality index $\mathscr{E}$, represented in terms of the function $\varphi(y)$ of equation 8 . The rows indicate whether the limit $\varphi(0):=\lim _{y \rightarrow 0} \varphi(y)$ is positive, or not. The columns indicate whether the limit $\varphi(\infty):=\lim _{y \rightarrow \infty} \varphi(y)$ is smaller than -2 , or not. The two rows and the two columns determine the four different scenarios, and for each scenario the equality index $\mathscr{E}$ is specified: the ratio $\mathscr{R}$, the ratio $\mathscr{R}_{l}$, the ratio $\mathscr{R}_{r}$, or zero.

\begin{tabular}{|l|c|c|}
\hline \hline & $\varphi(\infty)<-2$ & $\varphi(\infty) \geqslant-2$ \\
\hline$\varphi(0)>0$ & $\mathscr{R}=\frac{\varphi^{-1}(0)}{\varphi^{-1}(-2)}$ & $\mathscr{R}_{l}=\frac{\varphi^{-1}(0)}{\varphi^{-1}(-1)}$ \\
\hline$\varphi(0) \leqslant 0$ & $\mathscr{R}_{r}=\frac{\varphi^{-1}(-1)}{\varphi^{-1}(-2)}$ & 0 \\
\hline \hline
\end{tabular}

\section{Application}

Table 2 facilitates the wide application of the scenario-based equality index $\mathscr{E}$. Indeed, assume that we are given a positive-valued random variable $Y$, with probability density function $\psi(y)(0<y<\infty)$, to which we want to apply the scenario-based equality index $\mathscr{E}$. The first step is to check whether the underlying Langevin setting - with a U-shaped potential landscape - applies to the given random variable $Y$. Namely: is the underlying force $F(x)$ a continuous function that decreases monotonously from a positive limit $F(-\infty):=\lim _{x \rightarrow-\infty} F(x)>0$ to a negative limit $F(\infty):=\lim _{x \rightarrow \infty} F(x)<0$ ? Equation $(9)$ implies that this question, in terms of the function $\varphi(y)$ of equation (8), translates to the following question: is $\varphi(y)$ a continuous function that decreases monotonously from the limit $\varphi(0):=\lim _{y \rightarrow 0} \varphi(y)>-1$ to the $\operatorname{limit}$ $\varphi(\infty):=\lim _{y \rightarrow \infty} \varphi(y)<-1$ ? If the answer to the latter question is affirmative, then the scenario-based equality index $\mathscr{E}$ is applicable indeed, and we may proceed to the second step: computing this index, via the function $\varphi(y)$ of equation [8, according to table 2 
At the beginning of section 5 we noted that equality indices are required to be scale invariant. Equality indices were originally devised in order to gauge the socioeconomic equality of wealth distributions in human societies. Evidently, an equality score should be invariant with respect to the specific currency via which wealth is measured. Indeed, the same human society has the same degree of socioeconomic equality - no matter in which currency the wealth of the society members is measured. In the context of wealth distributions, the random variable $Y$ represents the wealth of a randomly sampled society member, and a change of currency is manifested by the following scale transformation: $Y \rightarrow c Y$, where $c$ is a positive constant. In turn, equality indices should be invariant with respect to this scale transformation. The scenario-based equality index $\mathscr{E}$ indeed satisfies the scale-invariance property: table 2 that corresponds to the scaled random variable $c Y$ is identical to table 2 that corresponds to the random variable $Y$.

Another transformation we address is reciprocation: $Y \rightarrow 1 / Y$. On the log-scale, $X=\ln (Y)$, reciprocation manifests mirroring: $X \rightarrow-X$. Reciprocation switches between the roles of the small "poor" values and the large "rich" values. The effect of reciprocation on the scenario-based equality index $\mathscr{E}$ is rather "elegant": table 2 that corresponds to the reciprocated random variable $1 / Y$ is given by the transposition of table 2 that corresponds to the random variable $Y$.

Yet another transformation we address is the power transformation: $Y \rightarrow Y^{p}$, where $p$ is a positive power. The power transformation is prevalent across the sciences, and the scenario-based equality index $\mathscr{E}$ that corresponds to the power random variable $Y^{p}$ is presented in table 3 . Note that for the power $p=1$ table 3 indeed yields back table 2

Table 3. The scenario-based equality index $\mathscr{E}$ of the power random variable $Y^{p}$, represented in terms of the function $\varphi(y)$ of equation 8 . The rows indicate whether the limit $\varphi(0):=\lim _{y \rightarrow 0} \varphi(y)$ is greater than $p-1$, or not. The columns indicate whether the limit $\varphi(\infty):=\lim _{y \rightarrow \infty} \varphi(y)$ is smaller than $-p-1$, or not. The two rows and the two columns determine the four different scenarios, and for each scenario the equality index $\mathscr{E}$ is specified: the ratio $\mathscr{R}$, the ratio $\mathscr{R}_{l}$, the ratio $\mathscr{R}_{r}$, or zero.

\begin{tabular}{|c|c|c|}
\hline \hline & $\varphi(\infty)<-p-1$ & $\varphi(\infty) \geqslant-p-1$ \\
\hline$\varphi(0)>p-1$ & $\mathscr{R}=\left[\frac{\varphi^{-1}(p-1)}{\varphi^{-1}(-p-1)}\right]^{p}$ & $\mathscr{R}_{l}=\left[\frac{\varphi^{-1}(p-1)}{\varphi^{-1}(-1)}\right]^{p}$ \\
\hline$\varphi(0) \leqslant p-1$ & $\mathscr{R}_{r}=\left[\frac{\varphi^{-1}(-1)}{\varphi^{-1}(-p-1)}\right]^{p}$ & 0 \\
\hline \hline
\end{tabular}

In sections 5 and 6 as well as in this section, we focused on the notion of equality. In fact, as noted in the introduction, it is more common to address inequality rather than equality. An inequality index $\mathscr{I}$ is a gauge of positive-valued random variables that scores their inherent inequality, i.e., their inherent statistical heterogeneity [27. 30 . Inequality indices take values in the unit interval, $0 \leqslant \mathscr{I} \leqslant 1$, yield the minimal zero score only in the case of constant random variables, and are scale-invariant (as described above). The shift between gauging equality and inequality is straightforward. Indeed, every equality in$\operatorname{dex} \mathscr{E}$ has a coupled inequality index $\mathscr{I}$ (and vice-versa), and the coupling is given by: $\mathscr{E}+\mathscr{I}=1$. Hence, all the results regarding the scenario-based equality index $\mathscr{E}$ can be shifted to the coupled scenario-based inequality index, $\mathscr{I}=1-\mathscr{E}$.

\section{Examples}

To demonstrate the application of the scenario-based equality index $\mathscr{E}$, we present in this section four illustrative examples. In all the examples we are given a positive-valued random variable $Y$ with probability density function $\psi(y)(0<y<\infty)$. In all the densities, the parameter $c$ denotes a normalization constant, and in the densities of examples 24 the parameters $\alpha$ and $\beta$ denote positive exponents.

Example 1

$$
\psi(y)=c \frac{1}{y} \exp \left\{-\frac{[\ln (y)-m]^{2}}{2 v}\right\} .
$$


This density characterizes the lognormal law with a real log-scale mean $m$ and a positive log-scale variance $v$ [79. 83]. Applying table 2]we obtain that

$$
\mathscr{E}=\mathscr{R}=\exp (-2 v)
$$

Namely, in this example only the scenario $\mathscr{E}=\mathscr{R}$ takes place.

\section{Example 2}

$$
\psi(y)=c \exp \left(-y^{\alpha}\right) y^{\beta-1} .
$$

This density generalizes the gamma law $(\alpha=1)[84]$ and the Weibull extreme-value law $(\alpha=\beta)[85$, 88]. Applying table 2 we obtain that

$$
\mathscr{E}=\left\{\begin{array}{lll}
\mathscr{R}=\left(\frac{\beta-1}{\beta+1}\right)^{1 / \alpha}, & \text { if } & \beta>1, \\
\mathscr{R}_{r}=\left(\frac{\beta}{\beta+1}\right)^{1 / \alpha}, & \text { if } & \beta \leqslant 1 .
\end{array}\right.
$$

Namely, in this example only the scenarios $\mathscr{E}=\mathscr{R}$ and $\mathscr{E}=\mathscr{R}_{r}$ take place.

\section{Example 3}

$$
\psi(y)=c \exp \left(-y^{-\alpha}\right) y^{-\beta-1} .
$$

This density generalizes the inverse-gamma law $(\alpha=1)$ [89.91] and the Fréchet extreme-value law $(\alpha=\beta)$ [92, 94. Applying table 2 we obtain that

$$
\mathscr{E}=\left\{\begin{array}{lll}
\mathscr{R}=\left(\frac{\beta-1}{\beta+1}\right)^{1 / \alpha}, & \text { if } & \beta>1, \\
\mathscr{R}_{l}=\left(\frac{\beta}{\beta+1}\right)^{1 / \alpha}, & \text { if } & \beta \leqslant 1 .
\end{array}\right.
$$

Namely, in this example only the scenarios $\mathscr{E}=\mathscr{R}$ and $\mathscr{E}=\mathscr{R}_{l}$ take place.

\section{Example 4}

$$
\psi(y)=c \frac{y^{\alpha-1}}{(1+y)^{\alpha+\beta}} .
$$

This density characterizes the beta-prime law, also known as the Fisher-Snedecor law 95, 97]. The scenario-based equality index $\mathscr{E}$ of this law is given in table 4

Table 4. The scenario-based equality index $\mathscr{E}$ corresponding to the beta-prime density of equation (16). The rows indicate whether the exponent $\alpha$ is greater than 1, or not. The columns indicate whether the exponent $\beta$ is greater than 1 , or not. The two rows and the two columns determine the four different scenarios, and for each scenario the equality index $\mathscr{E}$ is specified: the ratio $\mathscr{R}$, the ratio $\mathscr{R}_{l}$, the ratio $\mathscr{R}_{r}$, or zero.

\begin{tabular}{|c|c|c|}
\hline \hline & $\beta>1$ & $\beta \leqslant 1$ \\
\hline$\alpha>1$ & $\mathscr{R}=\frac{\alpha-1}{\alpha+1} \frac{\beta-1}{\beta+1}$ & $\mathscr{R}_{l}=\frac{\alpha-1}{\alpha} \frac{\beta}{\beta+1}$ \\
\hline$\alpha \leqslant 1$ & $\mathscr{R}_{r}=\frac{\alpha}{\alpha+1} \frac{\beta-1}{\beta}$ & 0 \\
\hline \hline
\end{tabular}

Several remarks regarding examples 24 First, the similarity between examples 2 and 3 is not coincidental: indeed, the random variables of these examples are related by reciprocation, $Y \rightarrow 1 / Y$, and hence the "transposition relation" of their respective scenario-based equality indices. Second, examples 2 and 3 can be obtained via the power transformation $Y \rightarrow Y^{1 / \alpha}$ where: the "original" random variable $Y$ is governed, respectively, by a gamma law with density $\psi(y)=c \exp (-y) y^{\beta / \alpha-1}$, and by an inverse-gamma 
law with density $\psi(y)=c \exp (-1 / y) y^{-\beta / \alpha-1}$. Third, note that in the case of example 4 with identical exponents, $\alpha=\beta$, only the scenarios $\mathscr{E}=\mathscr{R}$ and $\mathscr{E}=0$ take place.

A concluding note: this paper presented a novel Langevin-based method of scoring the statistical heterogeneity of a wide range of non-negative size distributions with both finite and infinite means - be they count, length, area, volume, mass, energy, duration, etc.; as this paper is dedicated to the $60^{\text {th }}$ birthday of Professor Yurij Holovatch, his students are most welcome to apply this method in the analysis of real-world data from the scientific fields of interest of Professor Holovatch - statistical physics, econophysics, and sociophysics - as well as from other fields of science.

\section{References}

1. Econophysics of Wealth Distributions, Chatterjee A., Yarlagadda S., Chakrabarti B.K. (Eds.), Springer, Milan, 2005.

2. Econophysics and Sociophysics: Trends and Perspectives, Chakrabarti B.K., Chakraborti A., Chatterjee A. (Eds.), Wiley, New York, 2006.

3. Chakrabarti B.K., Chakraborti A., Chakravarty S.R., Chatterjee A., Econophysics of Income and Wealth Distributions, Cambridge University Press, Cambridge, 2013.

4. Sen P., Chakrabarti B.K., Sociophysics: An Introduction, Oxford University Press, Oxford, 2014.

5. Buchanan M., Nat. Phys., 2014, 10, 471; doi 10.1038/nphys3016

6. Chatterjee A., In: Econophysics and Data Driven Modelling of Market Dynamics, Abergel F., Aoyama H., Chakrabarti B.K., Chakraborti A., Ghosh A. (Eds.), Springer, New York, 2015, 287-324.

7. Pareto V., Cours d'économie politique, Droz, Geneva, 1896 [Pareto V., Manual of Political Economy, reprint Edn., Oxford University Press, Oxford, 2014]

8. Meritocracy and Economic Inequality, Arrow K., Bowles S., Durlauf S.N. (Eds.), Princeton University Press, Princeton, 2000.

9. Social Inequality, Neckerman K.M. (Ed.), Russell Sage Foundation, New York, 2004.

10. Duclos J.Y., Araar A., Poverty and Equity: Measurement, Policy and Estimation with DAD, Springer, New-York, 2006.

11. Milanovic B., Worlds Apart: Measuring International and Global Inequality, Princeton University Press, Princeton, 2007.

12. Freeland C., Plutocrats: the Rise of the New Global Super-rich and the Fall of Everyone Else, Penguin Press, New York, 2012

13. Milanovic B., The Haves and the Have-Nots: A Brief and Idiosyncratic History of Global Inequality, Basic Books, New York, 2012.

14. Sutter J., Help Wanted: Must-Reads on Income Inequality and the Rich-poor Gap, CNN, 2013, August 19; http://edition.cnn.com/2013/08/19/opinion/sutter-inequality-must-reads/index.html.

15. Stiglitz J.E., The Price of Inequality: How Today's Divided Society Endangers Our Future, Norton, New York, 2013.

16. Deaton A., The Great Escape: Health, Wealth, and the Origins of Inequality, Princeton University Press, Princeton, 2013.

17. The Science of Inequality, a special issue of Science, 2014, 344, No. 6186.

18. The Economies of Rising Inequalities, Cohen D., Piketty T. (Eds.), Oxford University Press, Oxford, 2014.

19. Atkinson A.B., Inequality: What Can Be Done?, Harvard University Press, Boston, 2015.

20. Bourguignon F., The Globalization of Inequality, Princeton University Press, Princeton, 2015.

21. Stiglitz J.E., The Great Divide: Unequal Societies and What We Can Do About Them, Norton, New York, 2015.

22. Boix C., Political Order and Inequality, Cambridge University Press, Cambridge, 2015.

23. Piketty T., The Economics of Inequality, Harvard University Press, Boston, 2015.

24. Leopold L., Runaway Inequality, The Labor Institute Press, New York, 2015.

25. Milanovic B., Global Inequality, Harvard University Press, Boston, 2016.

26. Lindert P.H., Unequal Gains, Princeton University Press, Princeton, 2016.

27. Coulter P.B., Measuring Inequality: A Methodological Handbook, Westview Press, Boulder, 1989.

28. Advances on Income Inequality and Concentration Measures, Betti G., Lemmi A. (Eds.), Routledge, New-York, 2008.

29. Hao L., Naiman D.Q., Assessing Inequality, Sage, Los Angeles, 2010.

30. Cowell F., Measuring Inequality, Oxford University Press, Oxford, 2011.

31. Gini C., Variabilitá e mutabilita, 1912, reprinted in: Memorie di Metodologica Statistica, Pizetti E., Salvemini T. (Eds.), Libreria Eredi Virgilio Veschi, Rome, 1955.

32. Gini C., Econ. J., 1921, 31, 124; doi 10.2307/2223319 
33. Yitzhaki S., In: Research on Economic Inequality Vol. 8, Slottje D. (Ed.), Emerald Group Publishing Limited, Bingley, 1998, 13-30.

34. Yitzhaki S., Schechtman E., The Gini Methodology, Springer, New York, 2012.

35. Pietra G., Atti del Reale Istituto Veneto di Scienze, Lettere ed Arti, 1914-15, LXXIV, parte II, 775.

36. Hoover E.M., Rev. Econ. Stat., 1936, 18, 162; doi $10.2307 / 1927875$

37. Sarabia J.M., In: Modeling Income Distributions and Lorenz Curves, Chotikapanich D. (Ed.), Springer, New York, 2008, 167-190.

38. Eliazar I., Sokolov I.M., Physica A, 2010, 389, 117; doi 10.1016/j.physa.2009.08.006

39. Frosini B.V., Empir. Econ., 2012, 43, 175; doi 10.1007/s00181-011-0464-1

40. Sarabia J.M., Jorda V., Physica A, 2014, 416, 582; doi 10.1016/j.physa.2014.09.025

41. Eliazar I., Sokolov I.M., Physica A, 2012, 391, 1323; doi 10.1016/j.physa.2011.09.007

42. Eliazar I., Phys. Rep., 2016, 649, 1; doi 10.1016/j.physrep.2016.07.005

43. Lorenz M.O., Publ. Am. Stat. Assoc., 1905, 9, 209; doi 10.2307/2276207

44. Gastwirth J.L., Econometrica, 1971, 39, 1037; doi 10.2307/1909675

45. Giorgi G.M., Metron, 2005, 63, 299.

46. Arnold B.C., In: Advances on Income Inequality and Concentration Measures, Betti G., Lemmi A. (Eds.), Routledge, New-York, 2008, 12-24.

47. Modeling Income Distributions and Lorenz Curves, Chotikapanich D. (Ed.), Springer, New York, 2008.

48. Eliazar I., Physica A, 2015, 426, 93; doi 10.1016/j.physa.2015.01.016

49. Eliazar I., Physica A, 2015, 426, 116; doi 10.1016/j.physa.2014.12.021

50. Eliazar I., Chaos, Solitons Fractals, 2016, 92, 30; doi 10.1016/j.chaos.2016.08.012

51. Eliazar I., Investigating Equality: The Rényi Spectrum, 2016 (submitted).

52. Bouchaud J.P., Georges A., Phys. Rep., 1990, 195, 127; doi 10.1016/0370-1573(90)90099-N

53. Janicki A., Weron A., Simulation and Chaotic Behavior of Stable Stochastic Processes, Marcel Dekker, New York, 1994.

54. Samrodintsky G., Taqqu M.S., Stable Non-Gaussian Random Processes, Chapman \& Hall, New York, 1994.

55. Metzler R., Klafter J., Phys. Rep., 2000, 339, 1; doi 10.1016/S0370-1573(00)00070-3

56. Klafter J., Sokolov I.M., Phys. World, 2005, 18, 29; doi 10.1088/2058-7058/18/8/33.

57. Newman M.E.J., Contemp. Phys., 2005, 46, 323; doi 10.1080/00107510500052444

58. Redner S., A Guide to First-Passage Processes, Cambridge University Press, Cambridge, 2007.

59. Clauset A., Shalizi C.R., Newman M.E.J., SIAM Rev., 2009, 51, 661; doi $10.1137 / 070710111$

60. Nolan J.P., Stable Distributions: Models for Heavy Tailed Data, Birkhauser, New York, 2012.

61. Eliazar I., Klafter J., Phys. Rep., 2012, 511, 143; doi 10.1016/j.physrep.2011.11.001

62. Langevin P., C.R. Acad. Sci., 1908, 146, 530.

63. Coffey W.T., Kalmykov Yu.P., The Langevin Equation, 2nd Edn., World Scientific, Singapore, 2012.

64. Gardiner C., Handbook of Stochastic Methods, Springer, New York, 2004.

65. Van Kampen N.G., Stochastic Processes in Physics and Chemistry, 3rd Edn., North-Holland, Amsterdam, 2007.

66. Eliazar I., Cohen M.H., Phys. Rev. E, 2013, 88, 052104; doi 10.1103/PhysRevE.88.052104

67. Jaynes E.T., Phys. Rev., 1957, 106, 620; doi 10.1103/PhysRev.106.620.

68. Kapur J.N., Maximum-Entropy Models in Science and Engineering, Wiley-Interscience, New York, 1990.

69. Boltzmann L., Vorlesungen über Gastheorie Vol. I, II, Barth J.A., Berlin, 1896-1898.

70. Gibbs J.W., Elementary Principles in Statistical Mechanics, Yale University Press, New Haven, 1902.

71. Shannon C.E., Bell Syst. Tech. J., 1948, 27, 379; doi 10.1002/j.1538-7305.1948.tb01338.x

72. Lindenberg K., Shuler K.E., Seshadri V., West B.J., In: Probabilistic Analysis and Related Topics Vol. 3, BharuchaReid T. (Ed.), Academic Press, New York, 1983, 82-126.

73. Ramshaw J.D., Lindenberg K., J. Stat. Phys., 1986, 45, 295; doi 10.1007/BF01033092

74. Hull J.C., Options, Futures, and Other Derivatives, 6th Edn., Prentice-Hall, London, 2005.

75. Eliazar I., Klafter J., Physica A, 2006, 367, 106; doi 10.1016/j.physa.2005.11.026

76. Mantegna R.N., Stanley H.E., Introduction to Econophysics: Correlations and Complexity in Finance, Cambridge University Press, Cambridge, 2007.

77. Ito K., Mem. Am. Math. Soc., 1951, 4, 1.

78. Ito K., McKean H.P., Diffusion Processes and Their Sample Paths, Springer, Berlin, 1996.

79. Aitchison J., Brown J.A.C., The Lognormal Distribution with Special Reference to Its Uses in Econometrics, Cambridge University Press, Cambridge, 1957.

80. Lognormal Distributions: Theory and Applications, Crow E.L., Shimizu K. (Eds.), Marcel Dekker, New York, 1988.

81. Johnson N.L., Kotz S., Balakrishnan N., Continuous Univariate Distributions, Wiley, New York, 1994.

82. Limpert E., Stahel W., Abbt M., BioScience, 2001, 51, 341; doi 10.1641/0006-3568(2001)051\%5B0341:LNDATS\%5D2.0.CO;2 
83. Mitzenmacher M., Internet Math., 2004, 1, 226; doi 10.1080/15427951.2004.10129088

84. Feller W., Introduction to Probability Theory and Its Applications Vol. II, Wiley, New York, 1971.

85. Weibull W., Ingeniors Vetenskaps Akademiens Handligar, Stockholm, 1939, 151, 5.

86. Weibull W., ASME J. Appl. Mech., 1951, 18, 293.

87. Rinne H., The Weibull Distribution: A Handbook, CRC Press, Buca Raton, 2008.

88. Eliazar I., Phys. Rev. E, 2012, 86, 031103; doi 10.1103/PhysRevE.86.031103.

89. Berry D.A., Statistics: a Bayesian Perspective, Duxbury Press, Pacific Grove, 1995.

90. Bolstad W.M., Introduction to Bayesian Statistics, Wiley, New York, 2007.

91. Lee P.M., Bayesian Statistics: an Introduction, Wiley, New York, 2012.

92. Galambos J., Asymptotic Theory of Extreme Order Statistics, 2nd Edn., Krieger, Melbourne, Florida, 1987.

93. Kotz S., Nadarajah S., Extreme Value Distributions, Imperial College Press, London, 2000.

94. Reiss R.D., Thomas M., Statistical Analysis of Extreme Values: with Applications to Insurance, Finance, Hydrology and Other Fields, Birkhauser, Basel, 2007.

95. Iversen G.R., Norpoth H., Analysis of Variance, SAGE Press, Thousand Oaks, 1987.

96. Scheffé H., The Analysis of Variance, Wiley, New York, 1999.

97. Hocking R.R., Methods and Applications of Linear Models: Regression and the Analysis of Variance, Wiley, New York, 2003

\title{
Досліджуючи нерівності: підхід Ланжевена
}

\author{
I. Еліазар \\ Наукова команда в галузі інновацій розумних пристроїв, Група з розробки нових пристроїв, Корпорація \\ Інтел, Якум, Ізраїль \\ Індекси нерівності є кількісними показниками, які вимірюють відхилення розподілу багатства в людських \\ суспільствах від "основного стану" чистого комунізму. Хоча індекси нерівності були розроблені для засто- \\ сування в соціоекономіці, вони виявилися ефективно застосовними в контексті загальних ненегативних \\ розподілів розмірів, таких як кількість, довжина, площа, об'єм, маса, енергія та тривалість. Індекси не- \\ рівності зазвичай базуються на понятті кривих Лоренца, що неявно припускають існування скінчених \\ середніх. Як наслідок, Лоренц-базовані індекси нерівності вилучаються з множини нескінчених середніх \\ від розподілів розмірів. В цій статті ми представляємо індекс нерівності, який базується на загалом аль- \\ тернативному підході Ланжевена. Ланжевен-базовані індекси нерівності вводяться, вивчаються і засто- \\ совуються до широкого діапазону ненегативних розподілів розміру як зі скінченими, так і з нескінченими \\ середніми.
}

Ключові слова: індекси нерівності, криві Лоренца, рівняння Ланжевена, густина Гіббса, індекс сценарій-базованої рівності 